\title{
Performance Measurements of a Wireless Internet Service Providing (WISP) System
}

\author{
Qutaiba I. Ali and Ahmed Z. Saeed Al-wattar \\ Computer Eng. Dept. / Mosul University/Iraq
}

\begin{abstract}
In this paper, a comprehensive practical test to a Wireless Internet Service Provider (WISP) system is presented. The structure of the system is investigated and its performance is measured for different time units ranging from a Day to a Year periods. The effect of the different parameters on the system behavior is determined and their contribution is detected. The goal of this study is to give a realistic picture to the behavior of such a system as a result of its users demands, network infrastructure and service management techniques.
\end{abstract}

Key word: Wireless Internet service Provider, Latency, Throughput, WLAN Delay, and User Behavior

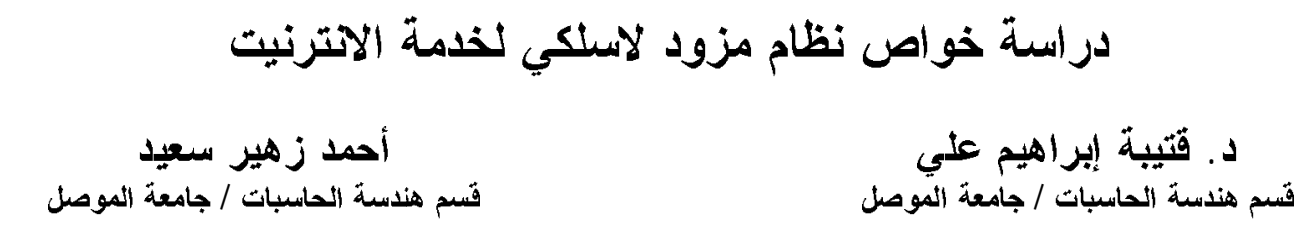

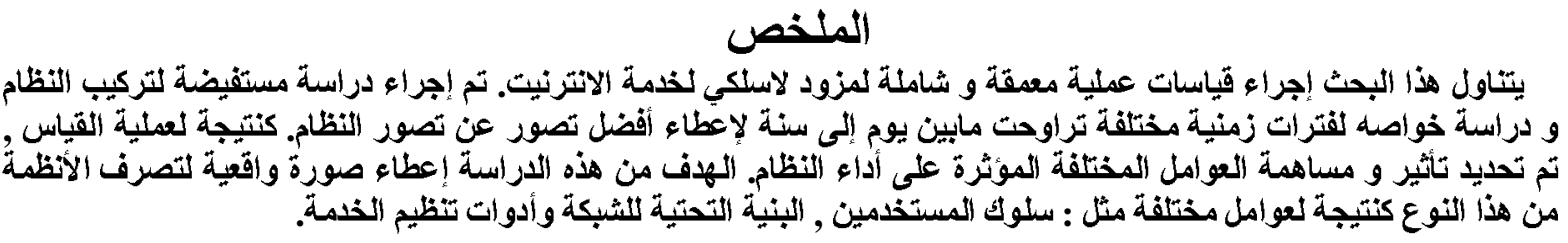




\section{INTRODUCTION}

Recently, Wi-Fi has become one of the most popular standards for wireless Internet access technology. Using radio frequency connections between a base station and devices with add-on or built-in 802.11 wireless cards, Wi-Fi gives access to the Internet and remote corporate and personal data without using the wires and cables of conventional wired networks in public places, homes, and offices. The global push to adopt 802.11 is based largely on its high bandwidth (up to $54 \mathrm{Mbps}$ ) and rich user experience that is comparable to being on a wired company LAN. This standard is open, unlicensed, internationally adopted, interoperable, and supported by every major player in the wireless LAN industry. WLAN options are available today for most consumer devices and the current technology of laptops, handheld PCs and PDAs are WLAN enabled [1]-[4].

There is a growing literature on wireless traffic measurement and Internet protocol performance over wireless networks. For example, Tang and Baker [5]-[6] discuss wireless network measurements from two different environments: a metropolitan area network, and a local area network. Balachandran et al. [7] report on network performance and user behavior for general Internet access by several hundred wireless LAN users during the ACM SIGCOMM conference in San Diego in 2001. They find that for this set of technology-literate users a wide range of Internet applications are used, user behaviors are diverse, and overall bandwidth demands are moderate. Kotz and Essein [8] characterize campus-wide wireless network usage at Dartmouth College, focusing on infrastructure mode using access points. G. Bai et al. [9] focuses on the performance of standalone wireless Web servers in short-lived wireless ad hoc networks. M. Narbutt et al. [10] experimentally investigates the relationship between resource utilization in WLANs and the quality of VoIP calls transmitted over wireless medium. Specifically they evaluate how the amount of free bandwidth Influences transmission impairments (i.e. delay, loss, and jitter) and thus call quality.

Our paper extends the work given in the above references to present comprehensive measurements covering all aspects and parts of a wireless internet service providing system. The main contribution of this work relies on the long term measurements made during a year period (2006-2007). The data given here could be used by the planners, developers and researchers of such a sophisticated network field.

\section{SYSTEM DESCRIPTION}

Internet services entered Iraq primarily at 1999. In the beginning, it was subjected to the government control and has limited services and speed. The main Internet distribution technology was the telephone wire modems with very low bit rate (under $1 \mathrm{kbps}$ with high noise \& attenuation) and hence downloads speed. After 2003, many private companies began to establish their own Internet service providers based on connecting the Internet through Satellite modems and wireless LAN (IEEE802.11) distribution network. The WISP system under study is considered the major internet service provider in the city of Mosul/Iraq. Its establishment began in the second half of 2003 and witnesses a wide expansion, in terms of coverage area and number of users, since then. The system gave various services to its clients, such as, Internet browsing, Email services, web hosting, HTML pages design, distributed gaming and numerous commercial activities. 
From the technical point of view, home Internet services providing is considered to be the major activity of the system. It used multiple wireless/wired network techniques to cover the different areas of the city. Fig. (1) Gives a clear picture of the network

a)

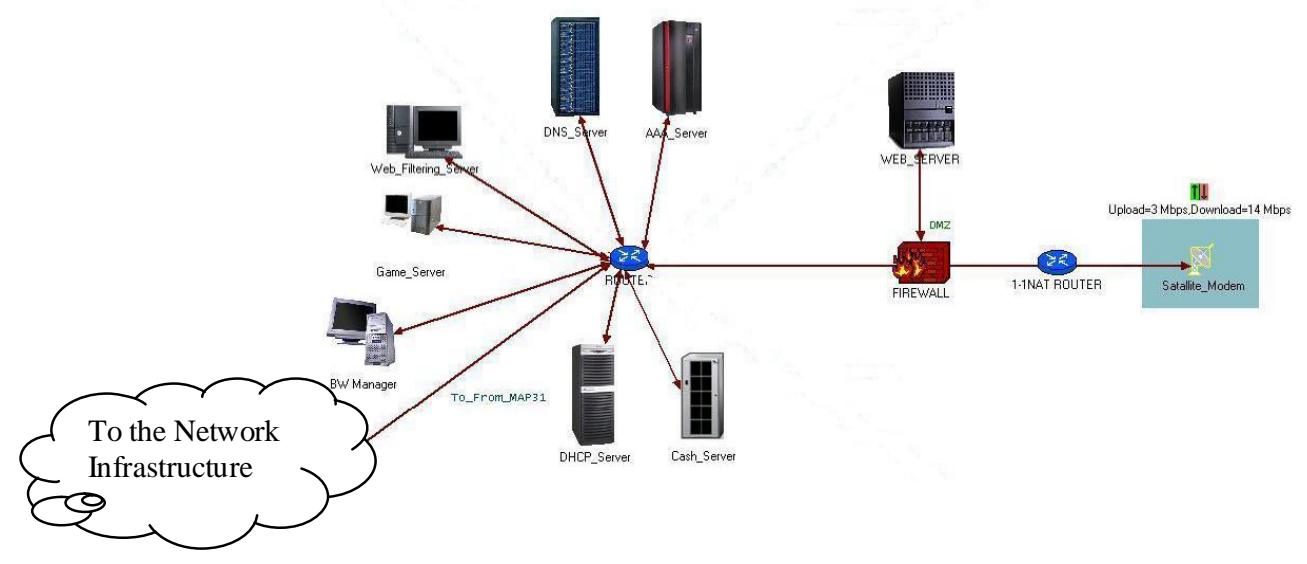

(b)

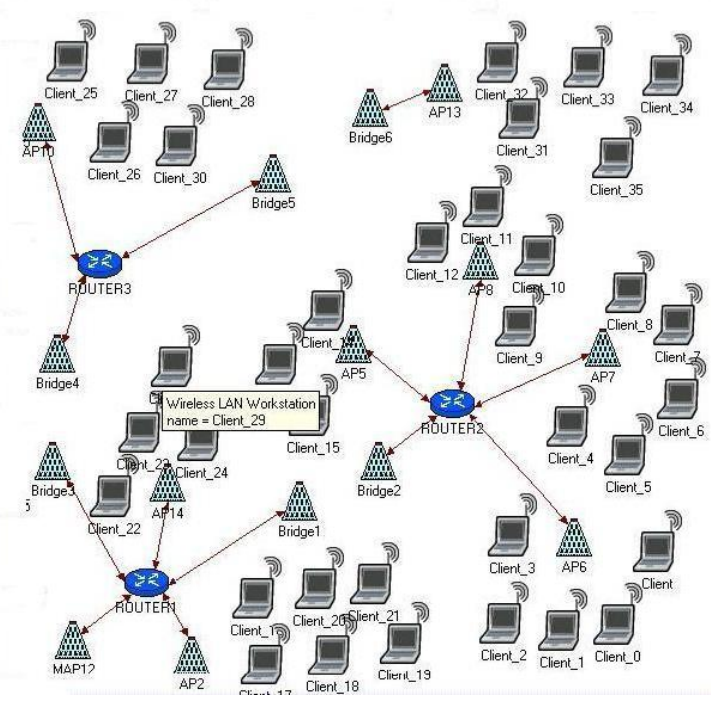

(c)

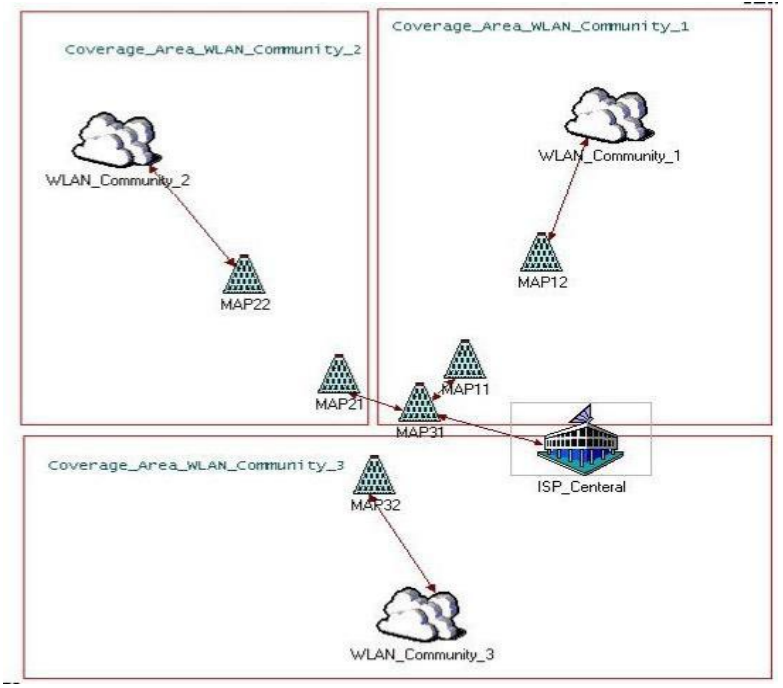

Fig. (1): Structure of WISP System: (a) ISP (Management) Center (b) Distribution Network (c) The Whole System

The network spans about $(25 \times 25 \mathrm{Km})$ of the city area. The major elements of the system are the distribution network and the ISP center.

- The distribution network: It is the various links between the clients and the ISP center. The traffic to/from the center is forwarded through three pairs of point to point bridges, we called Major Access points (MAP); each one of them is responsible of covering certain areas of the city. The wireless links between MAPs are subjected to IEEE802.11g WLAN standard, running at a speed of $(22 \mathrm{Mbps})$. It is worthwhile to mention that two of the MAPs are connected through a 
Fast Ethernet LAN to the third MAP, which is the gateway between the WLAN communities and the ISP center. The rest of the network consists of local access points (working at $11 \mathrm{Mbps}$ data rate of IEEE $802.11 \mathrm{~b}$ WLAN standard) installed in different sections of the city to serve certain number of users. The local access points were segmented into different VLAN groups to prevent inter-traffic between them. Fig. (2) Shows the installed access points with their relative users.

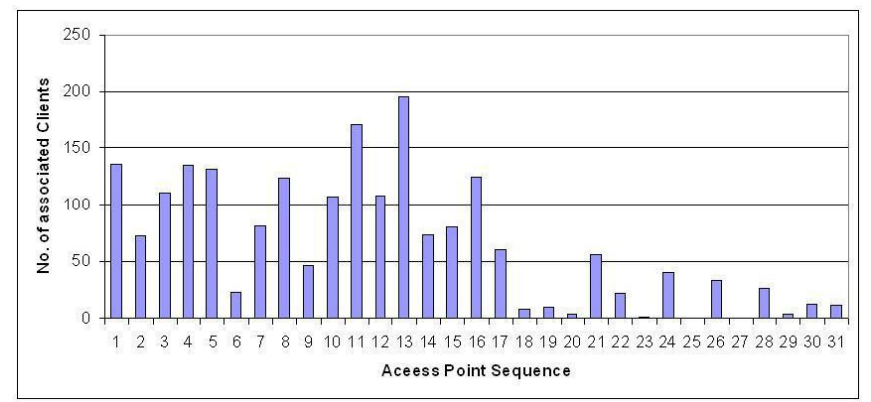

Fig. (2): Installed access points with their relative users.

It is clear that local access points have different number of users with an average of (63 client/AP). When the number of clients exceeds certain limit, additional access points are installed to serve the growing number of users. The coverage area of each access point is maintained through the use of proper antennas and if necessary, suitable signal boosters.

- The ISP Center: It consists of various network devices and servers to manage and provide internet services. Their functions could be summarized as follows:

- Satellite Modem: It is the link between the ISP and the rest of the internet. It has a data rates of (15 Mbps) for download and (3 Mbps) for upload operations.

1-1 NAT Router: The system has an (1500) public IP addresses pool available to the clients on the one to one Network Address Translation basis (i.e., each public IP is given to a private IP address during user log in time).

- A Firewall supported with Intrusion Detection System (IDS) capabilities provides certain level of security to the system. The company's web server is connected to the demilitarized zone (DMZ) portion of the firewall device. The firewall allows limited access to the DMZ, but because the DMZ only includes the public servers, an attack there only affects the servers and does not affect the other inside networks [3].

-AAA Server: AAA is the acronym for Authentication, Authorization, and Accounting. Authentication controls access by requiring valid user credentials, which are typically a username and password. Authorization controls access per user after users authenticate. Accounting tracks traffic that passes through the security appliance, gives the ability to have a record of user activity [3].

- Web filtering server together with the cache server frees more bandwidth for the benefit of the most important needs. As known [4], these servers have a great influence on the system performance and our measurements shows that a Hit Ratio of 0.35 is achieved (i.e., $35 \%$ of the traffic is acquired from the cache server rather than from the internet). On the other hand, filtering server frees $40 \%$ of the available bandwidth from the less important applications. Its decision is based on Content plus Database filtering algorithms according to pornography, spyware and viruses categories.

- Game server provides distributed game network between network clients. 
- Bandwidth Management Server: It is responsible for giving a certain level of service to the different clients according to their individual subscriptions. Each client is given a certain amount of bandwidth using several bandwidth management techniques. Also, many subscriptions have a pre defined amount of transferred data per month. Table (1) lists the various types of subscriptions.

TABLE (1): SUBSCRIPTION TYPES

\begin{tabular}{|c|c|}
\hline Subscription Type & Number of Clients \\
\hline $1.2 \mathrm{~GB} 64 \mathrm{~Kb} / \mathrm{s}$ Home $1-\mathrm{PC}$ & 205 \\
\hline 1.2 GB $64 \mathrm{~Kb} / \mathrm{s}$ Home 2-PC & 82 \\
\hline 1.2 GB $64 \mathrm{~Kb} / \mathrm{s}$ Home 3-PC & 160 \\
\hline $2.4 \mathrm{~GB} 64 \mathrm{~Kb} / \mathrm{s}$ Home 1-PC & 399 \\
\hline 2.4 GB $64 \mathrm{~Kb} / \mathrm{s}$ Home 2-PC & 146 \\
\hline 2.4 GB $64 \mathrm{~Kb} / \mathrm{s}$ Home 3-PC & 292 \\
\hline 3 GB $160 \mathrm{~Kb} / \mathrm{s}$ Home 1-PC & 219 \\
\hline 6 GB $160 \mathrm{~Kb} / \mathrm{s}$ Home 1-PC & 9 \\
\hline 9 GB $160 \mathrm{~Kb} / \mathrm{s}$ Home 1-PC & 1 \\
\hline $6 \mathrm{~GB} 256 \mathrm{~Kb} / \mathrm{s}$ Home 1-PC & 2 \\
\hline $1.5 \mathrm{~GB} 160 \mathrm{~Kb} / \mathrm{s}$ Home-Hours 1-PC & 24 \\
\hline 3 GB 160 Kb/s Home-Hours 1-PC & 213 \\
\hline $64 \mathrm{~Kb} / \mathrm{s}$ Café-IP-Unlimited & 5 \\
\hline $128 \mathrm{~Kb} / \mathrm{s}$ Cafe-Band & 23 \\
\hline $160 \mathrm{~Kb} / \mathrm{s}$ Cafe-Band & 48 \\
\hline $192 \mathrm{~Kb} / \mathrm{s}$ Cafe-Band & 8 \\
\hline $224 \mathrm{~Kb} / \mathrm{s}$ Cafe-Band & 5 \\
\hline $256 \mathrm{~Kb} / \mathrm{s}$ Cafe-Band & 1 \\
\hline $600 \mathrm{MB} 64 \mathrm{~Kb} / \mathrm{s}$ Home 2-PC & 1 \\
\hline $600 \mathrm{MB} 64 \mathrm{~Kb} / \mathrm{s}$ commercial 1-PC & 51 \\
\hline $600 \mathrm{MB} 64 \mathrm{~Kb} / \mathrm{s}$ commercial 2-PC & 11 \\
\hline $600 \mathrm{MB} 64 \mathrm{~Kb} / \mathrm{s}$ commercial 3-PC & 18 \\
\hline $1.5 \mathrm{~GB} 64 \mathrm{~Kb} / \mathrm{s}$ commercial 1-PC & 18 \\
\hline $1.5 \mathrm{~GB} 64 \mathrm{~Kb} / \mathrm{s}$ commercial 2-PC & 1 \\
\hline $1.5 \mathrm{~GB} 64 \mathrm{~Kb} / \mathrm{s}$ commercial 3-PC & 1 \\
\hline $1.5 \mathrm{~GB} 160 \mathrm{~Kb} / \mathrm{s}$ commercial 1-PC & 42 \\
\hline $3 \mathrm{~GB} 160 \mathrm{~Kb} / \mathrm{s}$ commercial 1-PC & 5 \\
\hline 4.5 GB $160 \mathrm{~Kb} / \mathrm{s}$ commercial 1-PC & 4 \\
\hline $3 \mathrm{~GB} 160 \mathrm{~Kb} / \mathrm{s}$ commercial 1-PC Full-time & 31 \\
\hline $6 \mathrm{~GB} 160 \mathrm{~Kb} / \mathrm{s}$ commercial 1-PC Full-time & 1 \\
\hline 9 GB $160 \mathrm{~Kb} / \mathrm{s}$ commercial 1-PC Full-time & 1 \\
\hline $3 \mathrm{~GB} 160 \mathrm{~Kb} / \mathrm{s}$ commercial-Hours 1-PC & 39 \\
\hline 3GB $160 \mathrm{~Kb} / \mathrm{s}$ Cafe-IP 5 to $9-\mathrm{PC}$ & 9 \\
\hline 3GB $160 \mathrm{~Kb} / \mathrm{s}$ Cafe-IP 10 to $14-\mathrm{PC}$ & 1 \\
\hline 3GB $160 \mathrm{~Kb} / \mathrm{s}$ Cafe-IP More than 14-PC & 19 \\
\hline $600 \mathrm{MB} 64 \mathrm{~Kb} / \mathrm{s}$ Home 1-PC & 5 \\
\hline Total Number of Clients & 2104 \\
\hline
\end{tabular}

-The WEB Server contains the web site of the company and hosted web sites for some individuals.

-At last, Domain Name Server(DNS) and Dynamic Host Configuration Protocol(DHCP) servers provide their usual tasks, such as translating IP addresses to a domain names (DNS function) and supplying the connected users with their corresponding IP addresses(DHCP function) [4].

The last issue to discuss in this section is the Service Level Agreement (SLA) of the system. It can be defined as the level of the services given to the user as given in the contract [6]-[7]. For the current system, SLA has the following criterion: 
-Average web page response time (Time needed to completely download a web page [8]) less than or equal to $(14 \mathrm{sec}$.) for $80 \%$ of the time.

-The bandwidth given to a user is full for $80 \%$ of the time.

- Average WLAN Latency $\leq 100$ msec.

$\bullet$ System (or network) failure rate (Availability) $\leq 12$ day/yea

\section{LOAD MEASUREMENTS}

In order to discover the real load applied to the system, a comprehensive load tests were made. The tests were implemented at different points on the network to give the maximum level of knowledge about the system. These points are: the satellite modem, content (http traffic) filter, game server, MAP1, MAP2, MAP3, a highly loaded local access point (AP4) and a lightly loaded access point (AP28). The data were collected for a year, month and day periods, as shown in Figs (3 to 5).
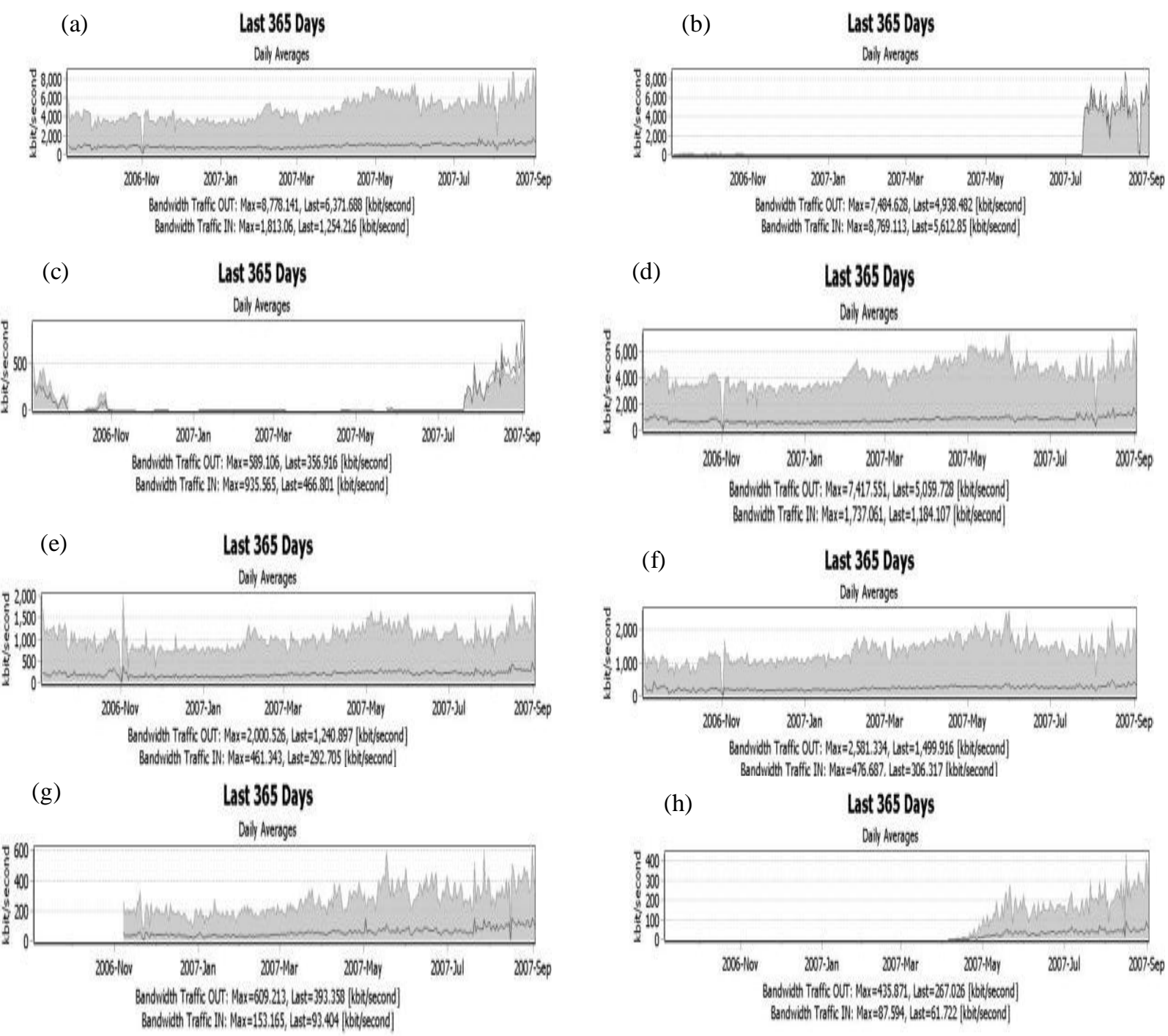

Fig.(3) :Yearly load of the system: (a)Satellite Modem (b)http filter (c)Game server (d)MAP3 (e)MAP2 (f)MAP1 (g)AP4 (h) AP28 
(a)

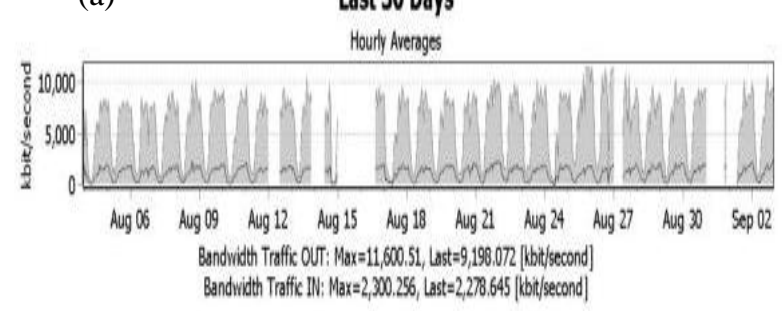

(c)

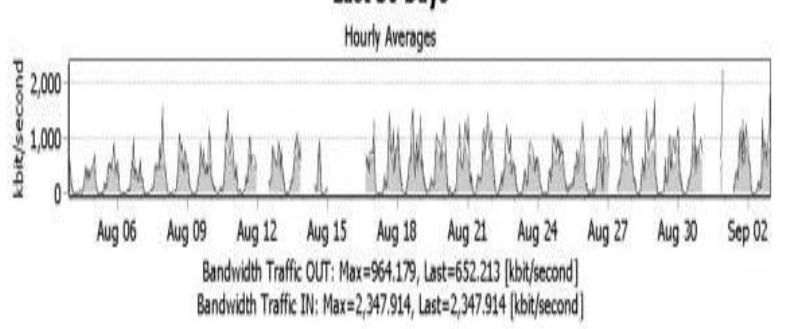

(e)

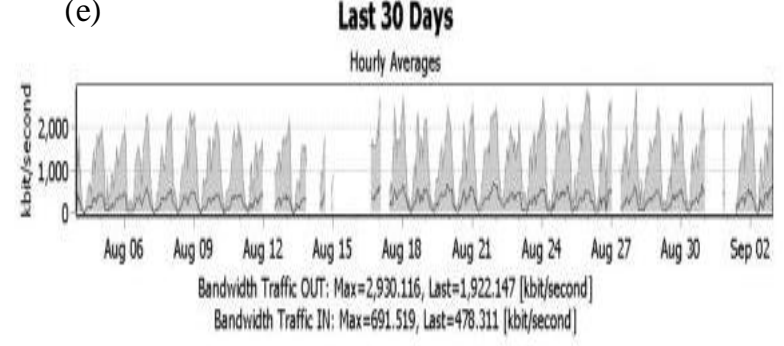

$(\mathrm{g})$

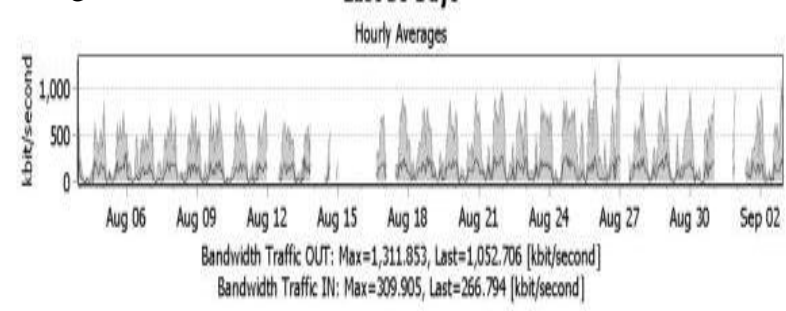

(b) Last 30 Days

Hount Arerages

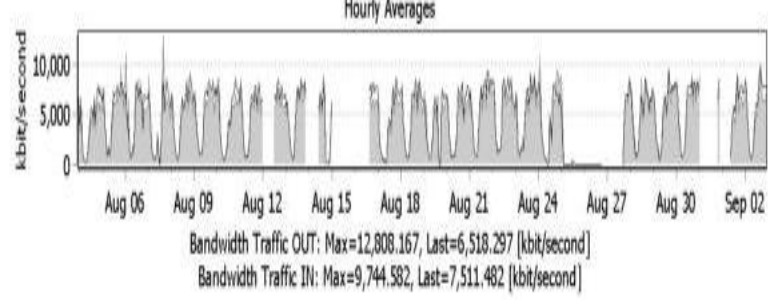

(d)

Last 30 Days

Houly Averages

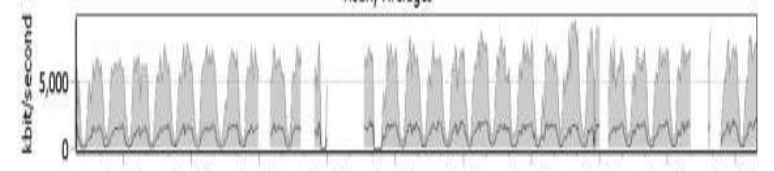

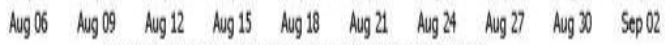

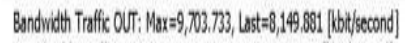

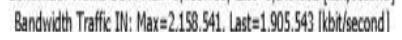

(f)

Last 30 Days

Howit Areages

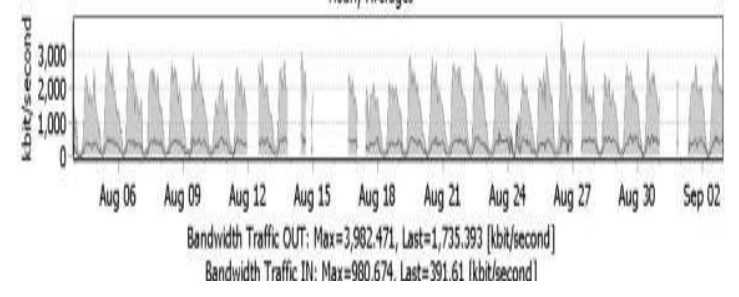

(h)

Last 30 Days

Hourty Arenges

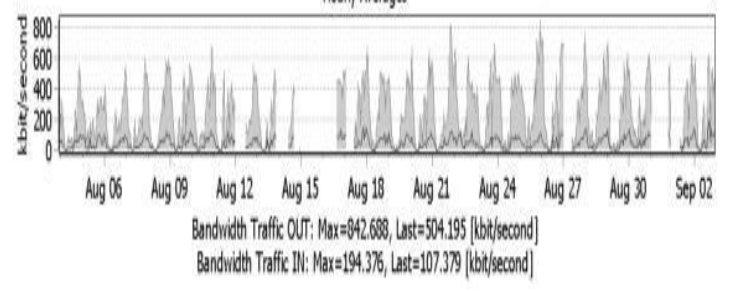

Fig.(4) :Monthly load of the system: (a)Satellite Modem (b)http filter (c)Game server (d)MAP3 (e)MAP2 (f)MAP1 (g)AP4 (h) AP28

(a)

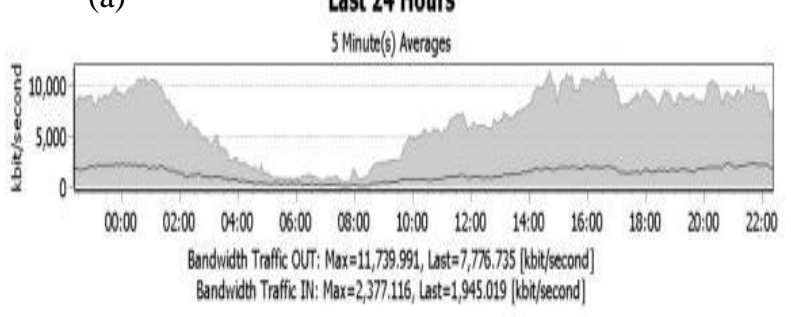

(b)

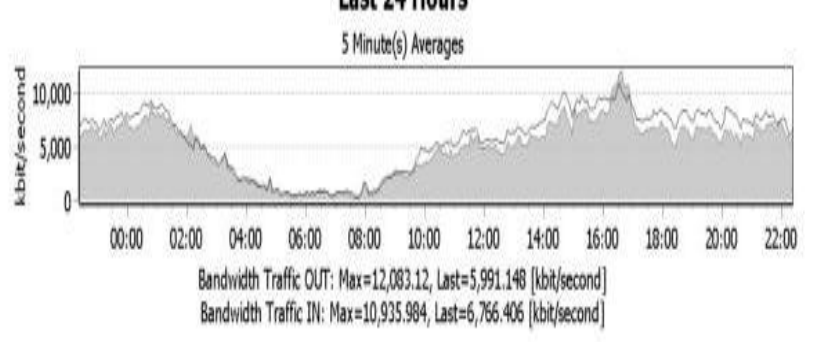


(c)

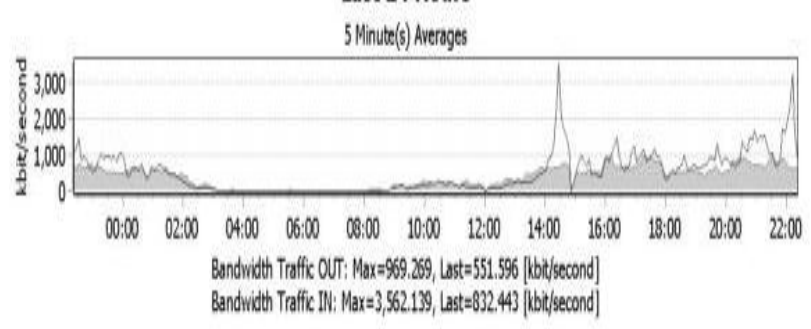

(e)

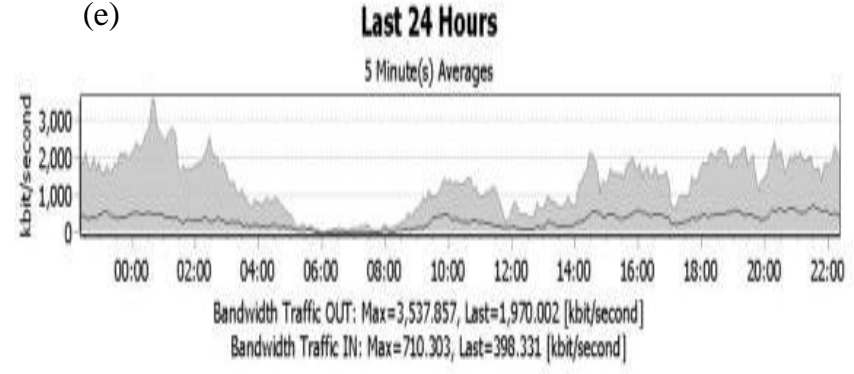

(g)

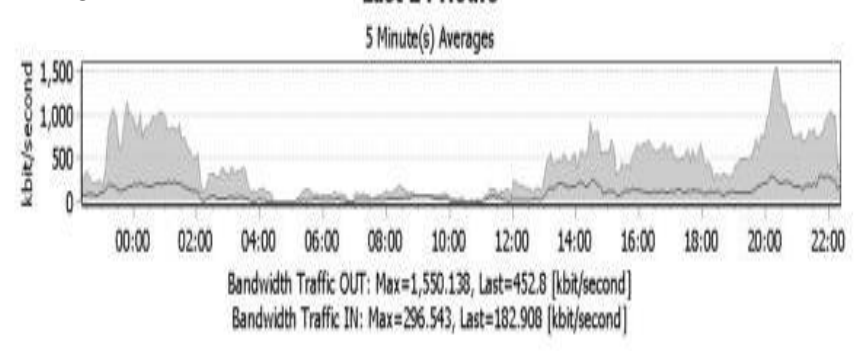

(d)

Last 24 Hours

5 Hinutes Areages

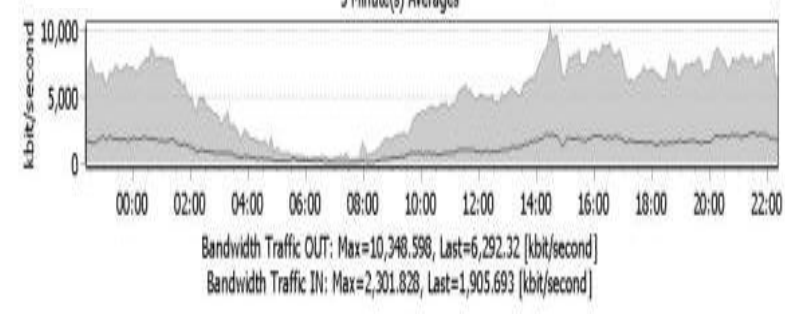

(f)

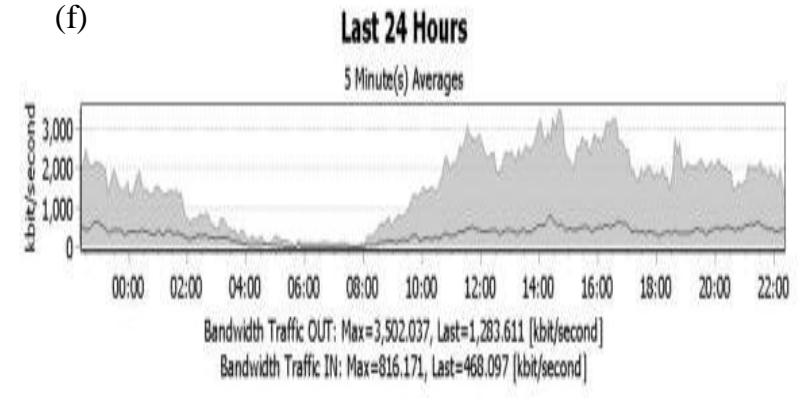

(h)

Last 24 Hours

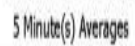

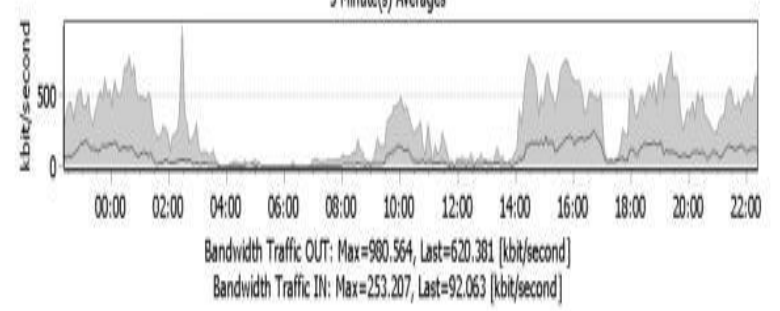

Fig.(5) :Daily load of the system(11/08/2007): (a)Satellite Modem (b)http filter (c)Game server (d)MAP3 (e)MAP2 (f)MAP1 (g)AP4 (h) AP28

The following remarks could be extracted from the above Figures:

1. The yearly average value of the downloaded traffic occupy (58.5\%) of the available (15 Mbps) bandwidth, while it consumes $(60 \%)$ of the available (3 Mbps) upload bandwidth. This result indicates the possibility of successful future expansion which is expected due to the load increment throughout the year.

2. Http traffic occupies (85\%) of the whole download bandwidth. Other applications are: file transfer applications (FTP), E-mail applications (SMTP), Domain Name Server Protocols (DNS) and Chatting protocols which share the remaining bandwidth.

3. The resultant traffic load applied to the system changes periodically between a low load period ( $16 \%$ of the available download bandwidth, from 4 to $10 \mathrm{am}$ ) and high load periods ( $78 \%$ of the available bandwidth) for the rest of the day.

4. The game server traffic witnesses a notable increase during the summer holiday as compared to other seasons. Also, a similar daily load distribution to that mentioned earlier is noticed here. 
5. The traffic directed from the infrastructure network is gathered via the three major access points and forwarded to the ISP center. In addition to the traffic received from the major access point (MAP3), ISP center receives an extra load from some local access points connected to it directly.

6. The average throughput of the local access points indicates an average user throughput of (4 kbps).

Not far from the above results is the statistical data gathered about the number of live (active) users of the system. This factor has a direct impact on the load applied to the network. Fig. (6) Illustrates the number of active users during different time units.

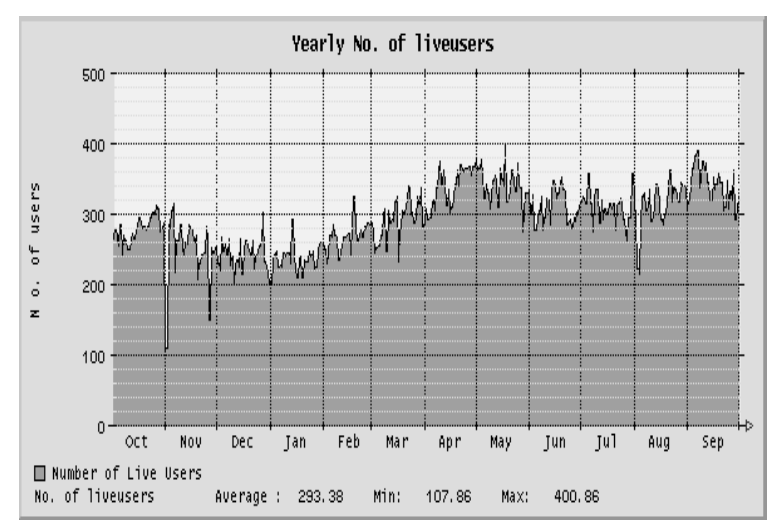

(a)

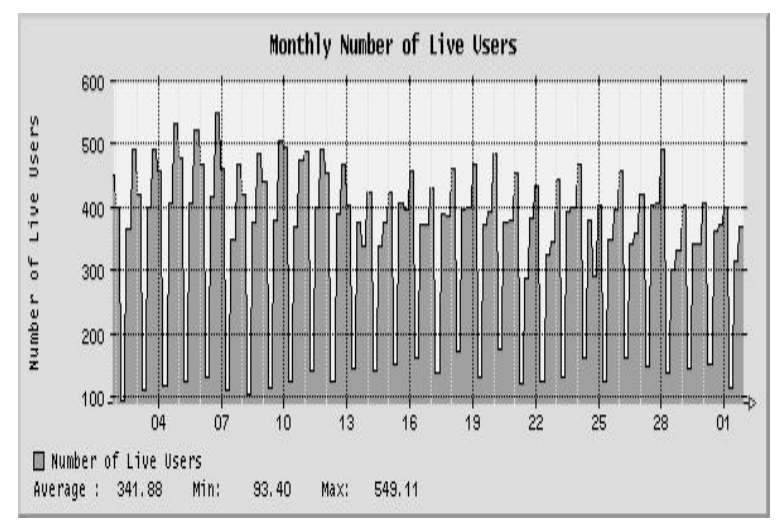

(b)

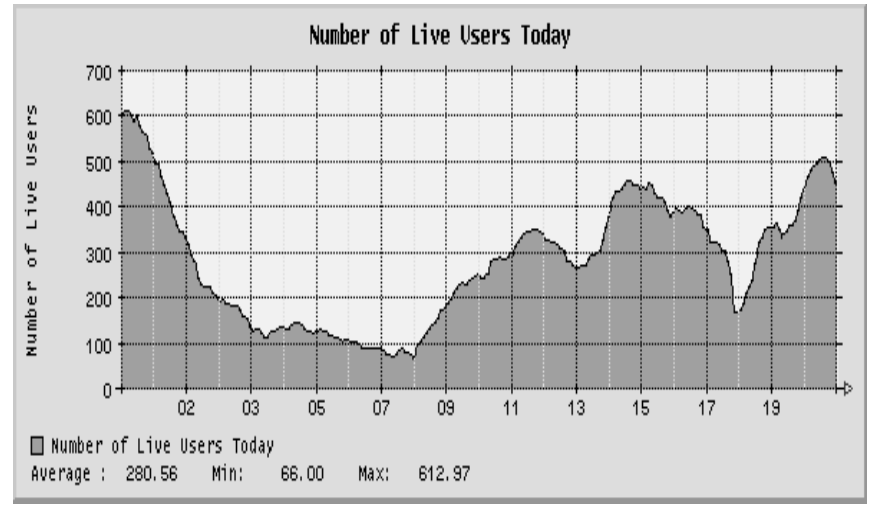

(c)

Fig. (6): Number of Live Users: (a) Yearly (b) Monthly (c) Daily

\section{LATENCY MEASUREMENTS}

In order to investigate the different contributors of the total network response time, system latency is measured. This is done using a PING command originating from different sources in the network to a certain server in the ISP centre during 3 days period, see Fig. (7).

The purpose of these experiments is to determine the real reason behind performance change in different situations. We chose two local access points, a high load access point (AP4) and a light load access point (AP28). These access points serve different number of clients and have 
comparable path lengths to the central ISP. Four latency measurements are done: from local access points to ISP centre (PING1 \& PING2), from a HOST to ISP center (PING3) and from ISP center to the internet (PING4). The latency values result from the different measurements are seen in Fig. (8).

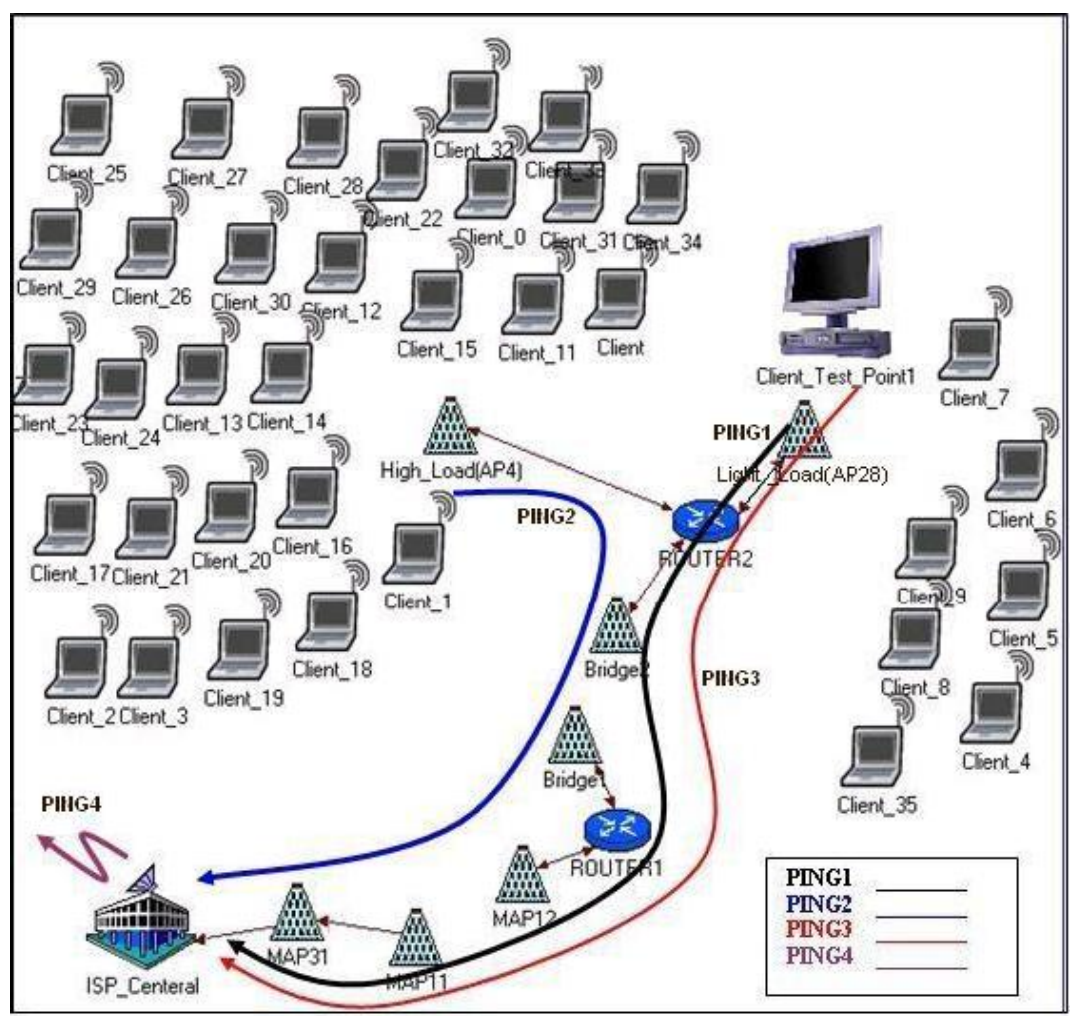

Fig. (7): Network Setup of Latency Measurements

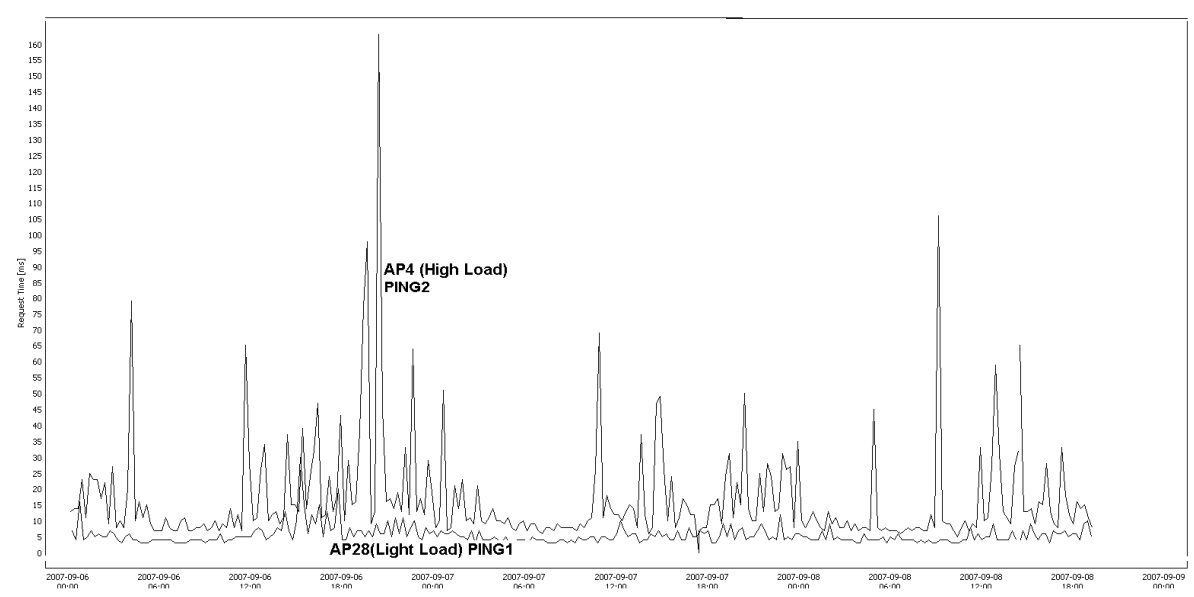

(a) 


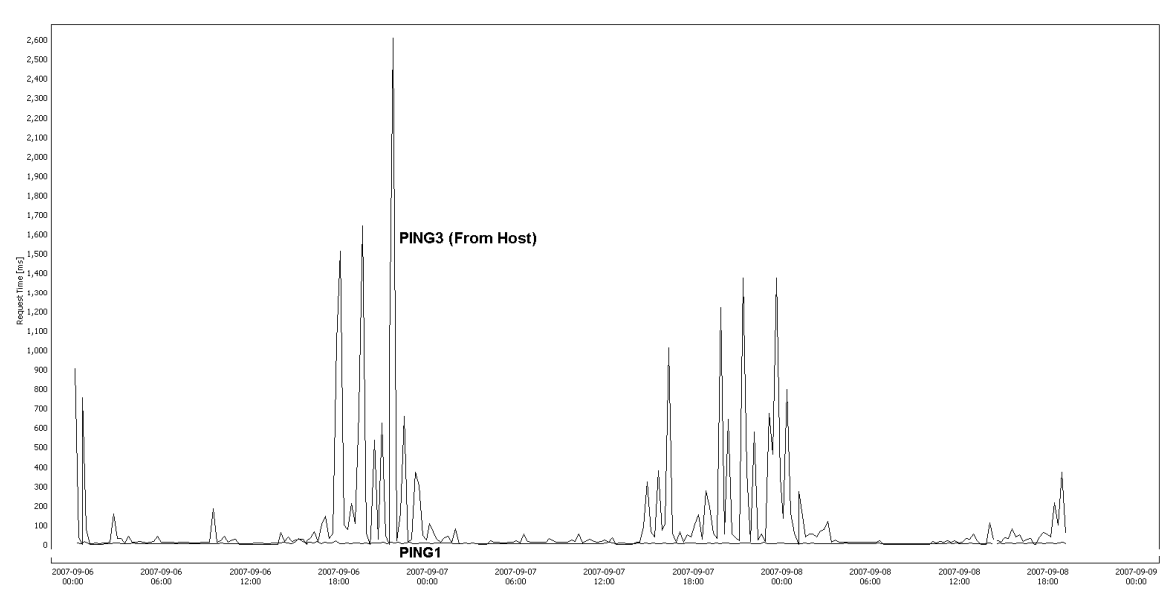

(b)

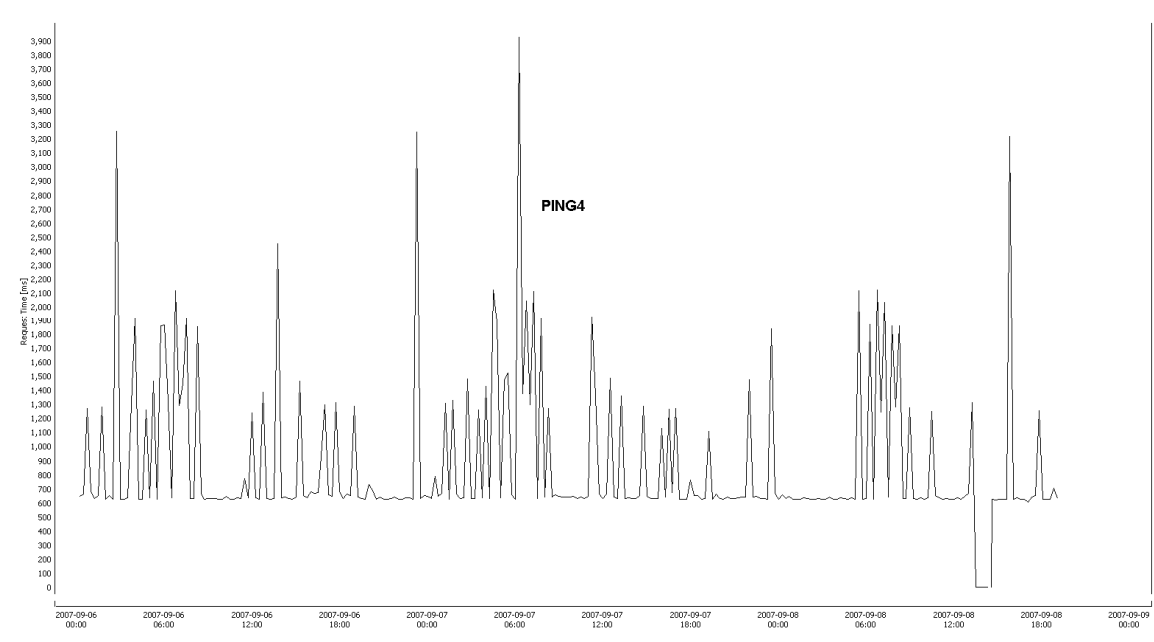

(c)

Fig. (8): Latency Measurements (a) PING1 \& PING2 (b) PING1\&PING3 (c) PING4

In these experiments, latency was measured as the summation of three parts:

1. from a client to the ISP center: One of the hosts is configured to send continues PING commands to the ISP center. Fig. ( $8 \mathrm{~b})$ shows that this latency has an average value of $(137 \mathrm{msec}$.) and subjects to the daily load distribution mentioned earlier. A comparison of this latency values to these of Ping1 (from access point to ISP centre) shows a higher contribution of this part of the network in the total latency budget. This is caused mainly by the contention nature of the CSMA/CA protocol (working in Distributed Coordination Point (DCF) mode) and its performance dependence on the number of served clients.

2. from local access point to the ISP center: The average values of this latency are $(5 \mathrm{msec}$.) for the lightly loaded access point (AP28) and (17 msec.) for the highly loaded access point (AP4), see Fig. (8a). It is obvious that this latency value is considered as a minor contributor and depends mainly on the number of contestant clients related to each access point. The relatively low latency in this section of the network indicates the successful planning of the installed distribution network. 
3. from ISP center to the Internet: We chose Yahoo server as the destination in this experiment because of its popularity (it was the Internet home page for most of the users). The average latency value in this case is $(885 \mathrm{msec}$.) and caused mainly by the effect of the propagation time to the geostationary satellite. The noted fluctuation in the latency results from various load conditions during test time, see Fig. (8c).

Total two way network latency could be calculated as the summation of both (ping3 and ping4) latencies and have the average value of $(1022 \mathrm{msec}$.).

To complete the picture, another test is done, page response time. Yahoo web site is chosen again to be the tested sample because of its popularity and the objects richness of its web pages. The purpose of this test is to assure system fulfillness to its service level agreement criteria mentioned earlier. The test procedure lasts for 24 hours and includes configuring one of AP4 (high load) clients to repeat the download operation of the page with and without the use of the cache server. The properties of the web page under test are listed in table (2) and the page response time is shown in Fig. (9).

TABLE (2): YAHOO WEB PAGE PROPERTIES

\begin{tabular}{ll}
\hline No. of Objects & 23 \\
No. of Image Objects & 17 \\
No. of Java Script Objects & 4 \\
No. of Style Sheet Documents & 1 \\
No. of Flash Objects & 1 \\
HTML Code Size(kBytes) & 133 \\
Total Page Size(kBytes) & 566 \\
\hline \hline
\end{tabular}

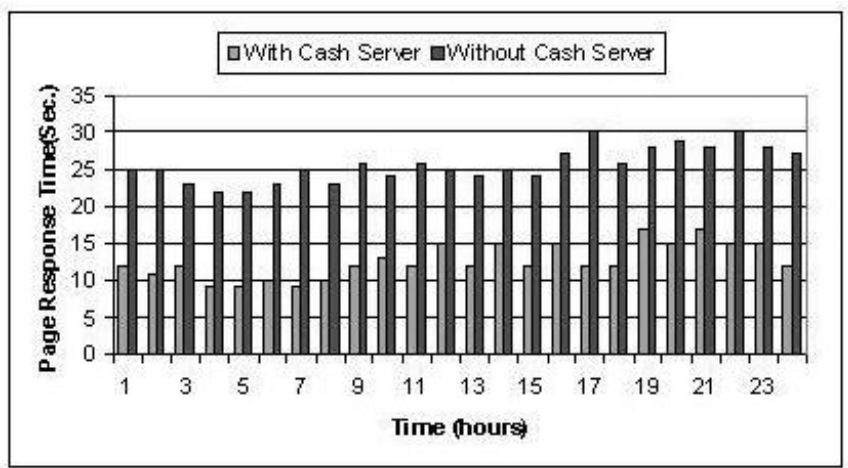

Fig. (9): Page Response Time Measurements

The average value of the page response time is $(12.6 \mathrm{Sec}$.) when using cache server and $(25.6$ Sec.) without it. This is a clear indication to the importance of web cache technique and its great influence on the system performance. Also, Fig.(9) assures the ability of the WISP system to respond successfully to the SLA criteria.

\section{INVESTIGATING USERS BEHAVIOR}

As shown in earlier, http was the major internet application and its traffic occupies most on the available bandwidth. This result gives a motive to discover the user behavior relating to this application. The first measure to be considered is the most visited web sites. In this manner, we used two metrics to evaluate a web site: the amount of the downloaded data from a site in a typical day and the number of TCP connections made to that web site. Tables ( 3 and 4 ) list the top 50 sites according to the above criteria. 
TABLE( 3): TOP 50 SITES WHICH REPRESENT 82\% OF THE DOWNLOADED DATA IN A ONE DAY PERIOD (11/08/2007)

\begin{tabular}{|c|c|}
\hline Accessed Site & Download Size(MByte) \\
\hline au.download.windowsupdate.com & 2000 \\
\hline msgr.dlservice.microsoft.com & 1000 \\
\hline us.js2.yimg.com & 754 \\
\hline 1.yimg.com & 480 \\
\hline us.i1.yimg.com & 444 \\
\hline mi.adinterax.com & 423 \\
\hline www.6rbtop.com & 401 \\
\hline$\underline{\text { ads.yimg.com }}$ & 386 \\
\hline us.dl1.yimg.com & 373 \\
\hline $\begin{array}{l}\text { mail.yimg.com } \\
\text { mimg }\end{array}$ & 367 \\
\hline insider.msg.yahoo.com & 334 \\
\hline www.google.com & 294 \\
\hline$\overline{\text { www.6rb.com }}$ & 289 \\
\hline pagead2.googlesyndication.com & 284 \\
\hline www.yahoo.com & 234 \\
\hline tbn0.google.com & 231 \\
\hline kh.google.com & 222 \\
\hline m1.2mdn.net & 208 \\
\hline mosul4all.com & 205 \\
\hline www.mosul4all.com & 199 \\
\hline $\begin{array}{l}\text { download.windowsupdate.com } \\
\end{array}$ & 198 \\
\hline www.microsoft.com & 153 \\
\hline f3.yahoofs.com & 151 \\
\hline 14.mihd.net & 148 \\
\hline$\overline{\text { d.yimg.com }}$ & 145 \\
\hline www.almawsil.com & 139 \\
\hline alrafidenland.com & 137.6 \\
\hline server2.mp3quran.net & 130 \\
\hline content.yieldmanager.edgesuite.net & 117 \\
\hline www.7shasha.com & 114 \\
\hline ad.z5x.net & 110 \\
\hline images.google.com & 100 \\
\hline pms.panet.co.il & 92 \\
\hline www.youtube.com & 90 \\
\hline www.aljazeera.net & 89.5 \\
\hline ads.adbrite.com & 82.6 \\
\hline www.bokra.net & 82 \\
\hline content.sweetim.com & 79 \\
\hline$\overline{\text { messenger.yahoo.com }}$ & 74 \\
\hline ad.yieldmanager.com & 74 \\
\hline video.google.com & 71 \\
\hline$\overline{\text { mail.google.com }}$ & 70 \\
\hline www.6rbwow.com & 69 \\
\hline www.mosulalhob.net & 63 \\
\hline www.aljazeerasport.net & 63 \\
\hline img.youtube.com & 62 \\
\hline$\overline{\text { Www.graaam.com }}$ & 59.5 \\
\hline forum.amrkhaled.net & 56.6 \\
\hline www.google-analytics.com & 54 \\
\hline www.hawar11.com & 50 \\
\hline
\end{tabular}




\begin{tabular}{llll} 
Al-Rafidain Engineering & Vol.18 & No.4 & August 2010 \\
\hline
\end{tabular}

TABLE (4): TOP 50 SITES WHICH REPRESENT 80\% OF THE TCP CONNECTIONS IN A ONE DAY PERIOD(11/08/2007)

\begin{tabular}{|c|c|}
\hline Accessed Site & No. of TCP connections (Thousands) \\
\hline us.i1.yimg.com & 173.5 \\
\hline au.download.windowsupdate.com & 164 \\
\hline mosul4all.com & 133.6 \\
\hline us.bc.yahoo.com & 94.7 \\
\hline www.mosul4all.com & 78.6 \\
\hline$\overline{\text { msgr.dlservice.microsoft.com }}$ & 77.8 \\
\hline tbn0.google.com & 66 \\
\hline pagead2.googlesyndication.com & 60 \\
\hline ads.yimg.com & 50 \\
\hline www.google-analytics.com & 42.16 \\
\hline www.almawsil.com & 42.14 \\
\hline 1.yimg.com & 41 \\
\hline 14.mihd.net & 40 \\
\hline www.google.com & 39 \\
\hline www.7 shasha.com & 37 \\
\hline insider.msg.yahoo.com & 36 \\
\hline us.js2.yimg.com & 31 \\
\hline messenger.yahoo.com & 30 \\
\hline kh.google.com & 28 \\
\hline ad.yieldmanager.com & 27 \\
\hline 72.52.165.228 & 25 \\
\hline content.sweetim.com & 24 \\
\hline www.mosulalhob.net & 23.3 \\
\hline mail.opi.yahoo.com & 23 \\
\hline www.6rb.com & 22.7 \\
\hline www.yahoo.com & 21.42 \\
\hline ad.z5x.net & 21.39 \\
\hline chat.alfnaan.com & 21.16 \\
\hline www.youtube.com & 19.2 \\
\hline ad.doubleclick.net & 18 \\
\hline img.youtube.com & 17.3 \\
\hline shttp.msg.yahoo.com & 17 \\
\hline www.ratteb.com & 16.9 \\
\hline stc.msn.com & 15.8 \\
\hline www.6rbtop.com & 15.5 \\
\hline m1.2mdn.net & 15.4 \\
\hline content.yieldmanager.edgesuite.net & 15.3 \\
\hline mail.google.com & 14 \\
\hline chat1.alfnaan.com & 13.89 \\
\hline www.4arab.com & 13.53 \\
\hline address.yahoo.com & 13.53 \\
\hline www.aljazeera.net & 13.34 \\
\hline qlbe.doook.com & 13.1 \\
\hline WwW.clocklink.com & 13 \\
\hline host1.digichet.com & 12.8 \\
\hline m1.webstats.motigo.com & 12.56 \\
\hline 74.52 .117 .218 & 12.55 \\
\hline host7.digichet.com & 12.51 \\
\hline us.music1.yimg.com & 12.38 \\
\hline us.i1.yimg.com & 173.5 \\
\hline
\end{tabular}

The most visited web site is (au.download.windowsupdate.com). This web site accessed automatically (and occasionally) by the WINDOWS operating system for the software update process, other web sites are accessed according to users' demands. 
From the above statistics, a better look to the users' activities in a typical work day could be created, as listed in table (5) below.

TABLE (5): TYPICAL DAILY USERS ACTIVITIES

\begin{tabular}{ll}
\hline Average number of live users & 300 out of 2104 \\
Average downloaded data/day & $69.8 \mathrm{GByte}$ \\
Average downloaded data/user/day & $232.6 \mathrm{MByte} \sim(7 \mathrm{Gbyte} / \mathrm{Month})$ \\
Average number of TCP Connections/day & $4.77 \mathrm{M}$ \\
Average number of TCP connections/user/day & $15.9 \mathrm{~K}$ \\
Average log time/user & 10 hours \\
\hline \hline
\end{tabular}

\section{CONCLUSIONS \& SUGGESTIONS FOR FUTURE WORK}

This paper abstracts one year work on the test and measurements of a popular WISP system. The following notes could be extracted from the above statistics:

1. The accurate plan prior to build the infrastructure network enhances system performance and allows a smooth future expansion.

2. The main contributor in the WLAN latency budget is the delay between the clients and their associated access points. In order to get an acceptable performance, the number of clients should not exceed a certain limit (60 node).

3. Web browsing was the dominant internet application and occupies most of the downloaded bandwidth (exactly $85 \%$ ).

4. The performance of the system could be greatly enhanced using a proper cache server. This technique has influence on both optimizing the used bandwidth and minimizing page response time( reduction by 50\%). Also, Filtering server frees more bandwidth (exactly 40\%)to the profit of the most important applications.

5. Daily http traffic has a periodic nature, i.e., it varies between low (16\% of the full bandwidth) and high load (78\% of the full bandwidth) values according to the users demands. This behavior is a direct result to the number of active users in these times and it is reflected on the network latency variation.

In spite of the fact that the current WISP system responds successfully to its Service Level Agreement (SLA) criteria, it is still far away from the level expected as compared to the Internet services provided to users worldwide. It was found that Internet users in Iraq still suffer from performance degradation due to multiple factors such as, contention nature of the WLAN protocols governs Internet sharing task (CSMA/CA protocol working under DCF mode) and limited bandwidth-high propagation delay features of the satellite communication systems. This study suggests the following enhancement to the Internet system in Iraq:

1. Installing a high speed network (such as SONET or 10Gigabit Ethernet) covers all major Iraqi cities.

2. Connecting the suggested Iraqi WAN to the Internet Backbone through high speed optical fibers.

3. Internet sharing \& distribution inside each city could be accomplished using modern high speed wireless networking techniques such as WLAN(IEEE802.11g) with QoS \& PCF mode, 4'th Generation mobile system or WiMAX (IEEE802.16). 


\section{ACKNOWLEDGMENT}

We thank AL MEHRAB for Internet and communications for the facilities provided during the preparation of this study.

\section{REFRENCES}

[1] S. Dixit and R. Prasad," Wireless IP and Building the Mobile Internet", Artech House, 2002.

[2] R. Kikta, A.Michael and P. Courtney, "Wireless Internet Crash Course", McGraw-Hill Inc., 2002.

[3] J. Gutérrez, "Business Data Communications and Networking: A Research Perspective", IDeA Group Publishing, 2007.

[4] G. Camponovo, and D. Cerutti, "WLAN communities and Internet access sharing: a regulatory overview", International Conference on Mobile Business, 11-13 July 2005 Page(s):281 - 287.

[5] D. Tang and M. Baker, "Analysis of a Metropolitan Area Wireless Network", Proceedings of ACM MOBICOM, Seattle, WA, pp. 13-23, August 1999.

[6] D. Tang and M. Baker, "Analysis of a Local-Area Wireless Network", Proceedings of ACM MOBICOM, Boston, MA, pp. 1-10, August 2000.

[7] A. Balachandran, G. Voelker, P. Bahl, and P. Rangan, "Characterizing User Behavior and Network Performance in a Public Wireless LAN", Proceedings of ACM SIGMETRICS, Marina Del Rey, CA, pp. 195-205, June 2002.

[8] D. Kotz and K. Essein, "Analysis of a Campus-Wide Wireless Network", Proceedings of ACM MOBICOM, Atlanta, GA, September 2002.

[9] G. Bai ,K. Oladosu, Performance and Robustness Testing of Wireless Web Servers, M.Sc. Thesis, University of Calgary, September 2003.

[10] M. Narbutt and M. Davis, "Experimental investigation on VoIP performance and the resource utilization in 802.11b WLANs", Proceedings 2006 31st IEEE Conference on Local Computer Networks,

Nov. 2006 Page(s):397 - 403. 